Received October 20, 2017

Revised November 13, 2017 Accepted November 14, 2017

\author{
Corresponding author \\ Jaemin Lee, M.D., Ph.D. \\ Department of Anesthesiology and \\ Pain Medicine, Seoul St. Mary's \\ Hospital, College of Medicine, The \\ Catholic University of Korea, 222 \\ Banpo-daero, Seocho-gu, Seoul \\ 06591, Korea \\ Tel: 82-2-2258-2236 \\ Fax: 82-2-537-1951 \\ E-mail:jmlee@catholic.ac.kr \\ ORCID \\ http://orcid.org/0000-0002-0224-7141
}

\section{The implication of using dominant hand to perform laryngoscopy: an analysis of the laryngoscopic view and blade-tooth distance}

\author{
Serin Lee, Jaewon Huh, Jae Sang Lee, and Jaemin Lee
}

Department of Anesthesiology and Pain Medicine, Seoul St. Mary's Hospital, College of Medicine, The Catholic University of Korea, Seoul, Korea

Background: Existing laryngoscopes are designed to be handled by the left hand, whereas most healthcare professionals are right-handed. However, controlling the laryngoscope device requires considerable strength and refinement to control the blade. We examined the usefulness of a right-handed laryngoscope to validate its clinical applicability.

Methods: One hundred sixty-four patients for general anesthesia were involved. Laryngoscopy was performed twice for each patient, once using a conventional left-handed Macintosh No. 3 laryngoscope and once using a right-handed one, by 25 right-handed and 18 left-handed laryngoscopists. The perpendicular distance from the tip of the maxillary incisor to the flange of each blade was measured when the maximum visibility of the glottis was obtained. We compared the distances, chances of directly contacting the tooth, laryngoscopic views and subjective feeling of difficulty in handling device between the two laryngoscopes.

Results: For the right-handed laryngoscopists, distance varied significantly between the two laryngoscopes $(5.0 \pm 3.5$ and $5.7 \pm 3.7 \mathrm{~mm}$ [mean \pm standard deviation] for the conventional and right-handed laryngoscopes, respectively $[P<0.001])$. The righthanded laryngoscope was associated with a decreased chance of directly contacting the teeth $(P=0.001)$. Additionally, the right-handed laryngoscope provided a better view than the conventional one $(P=0.005)$. Conversely, most of the left-handed laryngoscopists felt that the procedure using a conventional laryngoscope was easier than with a right-handed one.

Conclusions: When a right-handed laryngoscopist uses a right-handed laryngoscope, a better laryngoscopic view and a reduced chance of blade contact with the teeth can be achieved.

Key Words: Laryngoscope, Laryngoscopic view, Left-handed, Right-handed, Tooth.

\section{INTRODUCTION}

Existing laryngoscopes used for tracheal intubation are required to be operated by the left hand, regardless of which hand the operator uses dominantly. Although most tools and apparatus used in daily life are designed differently for left- and right-handed users, medical instruments, particularly those that are important to the lives of the patients, such as a laryngoscope, are designed without such consideration. This irrational design is an issue that remains unresolved.

Additionally, although most healthcare professionals are right-handed, that laryngoscopes are designed to favor

This is an Open Access article distributed under the terms of the Creative Commons Attribution Non-Commercial License (http://creativecommons.org/licenses/by-nc/4.0) which permits unrestricted non-commercial use, distribution, and reproduction in any medium, provided the original work is properly cited. 
the use of the left hand raises certain issues. The process of tracheal intubation by conventional laryngoscopy entails the following: a view of the larynx is obtained by manipulating the laryngoscope with the left hand, and then a tube is pushed into the trachea with the right hand. This is a standard practice used in all medical institutions. Training for novice professionals is also provided based on this standard procedure [1]. One possible explanation would be that, when laryngoscopes were first invented, pushing in the tube was thought to be more important than controlling the laryngoscope itself; thus, the right hand was used to insert the tubes, while the other hand was used to control the device. Such practice continues to this day.

However, of these two actions (pushing in the tube and controlling the laryngoscope device), control of the device requires more strength and refinement. Pushing in the tube may be performed easily, without requiring specific skills or strength, once the glottis is well exposed as a result of the operation of the laryngoscope [2]. On the other hand, controlling the laryngoscope to expose the glottis adequately requires considerable strength to lift the lower chin and refined positioning of the blade of a laryngoscope. When the blade of the laryngoscope is precisely positioned on the vallecula with refined control of the laryngoscope, and the lower chin is lifted with a firm push upward, the laryngoscope can have maximum visibility, and pushing in the tube would be much easier. Additionally, the distance from the teeth to the blade of the laryngoscope could be maximized with the strength that lifts up the lower chin, minimizing the risk of damage to the teeth $[3,4]$. Therefore, it would be reasonable to allow the operator to use his/her right hand if he/she is right-handed and the left hand if he/she is left-handed to control the laryngoscope. For female healthcare professionals who are not as strong as their male counterparts, such adequacy would matter more. However, although reports exist regarding the usefulness of right-handed laryngoscopes during the training cases of one-armed anesthesiology residents or when there is a gigantic mass in the mouth [5-7], no systematic study of this issue has been conducted.

Thus, the authors examined the implication of using dominant hand to perform laryngoscopy and its clinical and educational applicability in terms of securing the laryngoscopic view and protecting the teeth by obtaining the maximum distance between the laryngoscope blade and teeth.

\section{MATERIALS AND METHODS}

This study protocol was approved by the Institutional Review Board (IRB no. KC13DISI0344) and was registered in the Clinical Research information service Republic of Korea

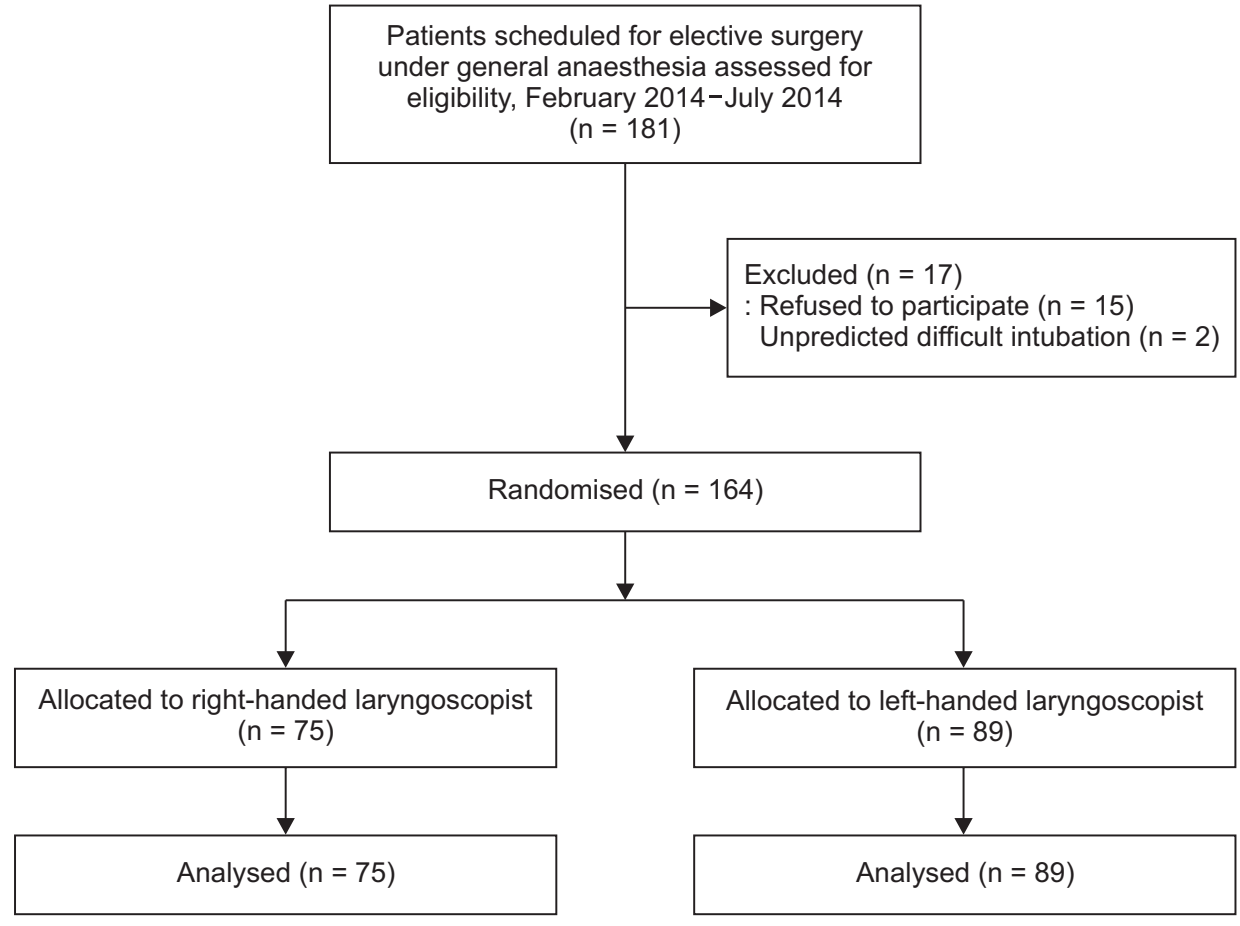

Fig. 1. The flow diagram of the patient population. 
(CRiS no. KCT0001154). Written informed consent was obtained from all patients before enrolment. We performed the present study from February 2014 to July 2014. The patient population was 164 American Society of Anesthesiologists physical status classification I and II patients, aged 18 years or older, scheduled for elective surgery requiring general anesthesia with tracheal tube placement. The patients excluded were those with cardiovascular disease, such as diabetes, hypertension, ischemic heart disease and arrhythmia, cervical spine fracture or cervical spine instability, patients with a history of gastroesophageal reflux, patients with traumatic facial abnormalities, and patients who were edentulous or wearing dentures (Fig. 1). Laryngoscopists were senior resident anesthesiologists, fellowships and faculty anesthesiologists; 25 right-handed and 18 left-handed. Resident anesthesiologists participated in our study while they were trained for 3 months in our hospital which is the main hospital among eight other affiliated hospitals where residents rotate every three months. Fellows and other staff members did not rotate and worked in our hospital throughout the study. Laryngoscope procedure for data collection was performed three to five times at monthly intervals to each laryngoscopist.

Before participating in the current study, each laryngoscopist performed approximately 10 practice rounds with the right-handed laryngoscope and a manikin (Laerdal Airway Trainer, Adult, Laerdal Medical, USA) to become familiar with the tool.

Before the induction of anesthesia, a standard hard sponge square pillow, about $10 \mathrm{~cm}$ in height, was placed under the head of each patient. Under the standard anesthetic monitoring, anesthesia was induced with propofol $1.5-2.0 \mathrm{mg} / \mathrm{kg}$, fentanyl 50-100 $\mu \mathrm{g}$, and rocuronium $1 \mathrm{mg} / \mathrm{kg}$ after de-nitrogenation with $100 \%$ oxygen. After the patient went to sleep, laryngoscopy procedures were performed twice for each patient, once using the conventional left-handed regular Macintosh No. 3 laryngoscope and once using the right-handed Macintosh No. 3 laryngoscope (SunMed, USA) (Fig. 2). The sequence of laryngoscope use was determined by a coin-toss method for each patient. When the laryngoscopist raised the laryngoscope and visibility of the glottis was obtained, the assistant measured the perpendicular distance from the tip of the maxillary central incisor to the flange of each blade (the blade-tooth distance) with a ruler, as previously described by Watanabe et al. [8]. We also recorded the cases in which

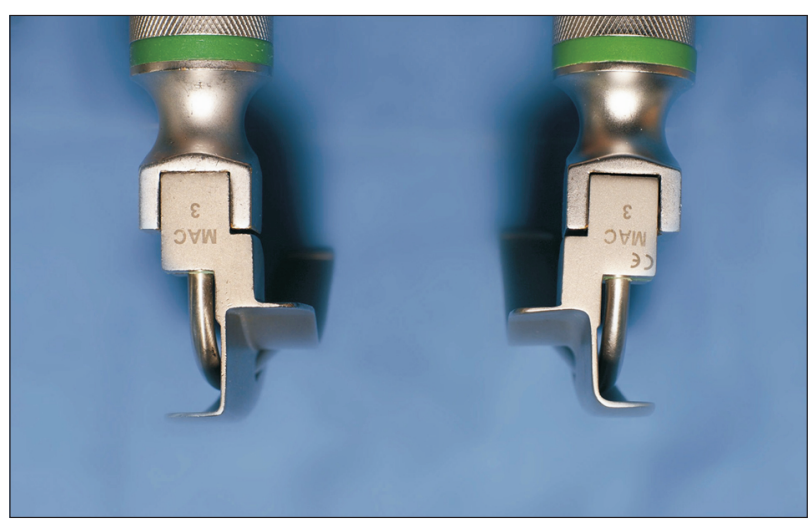

Fig. 2. Conventional left-handed regular Macintosh No. 3 laryngoscope (left) and right-handed Macintosh No. 3 laryngoscope (right) (SunMed, USA).

direct contact between the blade and upper tooth was inevitable during glottic exposure, causing the actual bladetooth distance to be $0 \mathrm{~mm}$. We asked the laryngoscopists to grade the view for each laryngoscopy. The best view obtained was noted according to Cormack and Lehane as follows: 1 . the whole vocal cords were visible; 2 . only the arytenoids or posterior commissure of the vocal cord was visible; 3 . only the epiglottis was visible; 4 . no glottic structure was visible [9]. External laryngeal pressure was applied when necessary, and whether the laryngeal maneuver was performed was noted. To eliminate the effect of the laryngeal maneuver on the laryngoscopic view and the blade-tooth distance, we measured them before the laryngeal maneuver was applied. Tracheal intubation was performed on the second laryngoscopy. The laryngoscopists were also asked to compare the difference between the two laryngoscopes for the subjective feeling of difficulty in handling the laryngoscopes.

From our previous observations, the mean blade-tooth distance when using the conventional left-handed laryngoscope was $2.0 \mathrm{~mm}$ with a standard deviation (SD) of $1.8 \mathrm{~mm}$ [3]. Assuming a $50 \%(=1.0 \mathrm{~mm})$ increase in the mean blade-tooth distance by the right-handed laryngoscope to be clinically significant, a minimum sample size of 53 patients was calculated for right-handed laryngoscopists, with a type I error of 0.05 and a power of 0.8 .

To analyze the difference in blade-tooth distances between the two types of laryngoscopes, paired $t$-test was used. The laryngoscopic view, frequencies of direct contact (i.e., bladetooth distance $=0 \mathrm{~mm}$ ), and subjective feeling of difficulty in handling the laryngoscope according to the different types 
of laryngoscopes were compared by Fisher's exact test. A P value less than 0.05 was deemed to indicate statistical significance.

\section{RESULTS}

Demographic data of the patients and the laryngoscopists are listed in Tables 1 and 2, respectively. No differences were found in the gender ratio and occupational status between right-handed and left-handed laryngoscopists.

For right-handed laryngoscopists, blade-tooth distances were 5.0 (3.5) $\mathrm{mm}$ and 5.7 (3.7) $\mathrm{mm}$ (mean [SD]) for the conventional and right-handed laryngoscope, respectively $(\mathrm{P}<$ 0.001). Dental contact occurred in $4 \%(3 / 75)$ of patients with the conventional laryngoscope versus $1.3 \%(1 / 75)$ with the right-handed laryngoscope $(\mathrm{P}=0.001)$. Conversely, the results were opposite for the left-handed laryngoscopists. The blade-tooth distance was decreased and frequency of direct contact between the blade and tooth was increased with the right-handed laryngoscope compared with the conventional laryngoscope $(\mathrm{P}<0.001$ and $\mathrm{P}=0.001$, respectively) (Table 3 ).

The results of laryngoscopic view were similar to those of the blade-tooth distance. For right-handed laryngoscopists, laryngoscopic views were significantly better with the righthanded laryngoscope $(\mathrm{P}=0.005)$. About $4 \%$ vs. $12 \%$ of cases involved a poor laryngoscopic view (grade III or IV) for the right-handed and conventional laryngoscope, respectively (Table 4$)$. About $30 \%(27 / 89 \times 100)$ of the laryngoscopies performed by left-handed laryngoscopists was associated with a worse laryngoscopic view by a right-handed laryngoscope than by a conventional laryngoscope; however, only $3 \%(2 / 75$ $\times 100)$ of the laryngoscopies performed by right-handed laryngoscopists confirmed that the laryngoscopic view worsened using a right-handed laryngoscope. About 23\% (17/75 $\times 100$ ) of the laryngoscopies performed by right-handed laryngoscopists showed that the right-handed laryngoscope provided a better laryngoscopic view than the conventional laryngoscope $(\mathrm{P}<0.001)$ (Table 5).

Most of the left-handed laryngoscopists felt that the procedures were much easier using the conventional laryngoscope, with nobody put right-handed laryngoscope above conventional laryngoscope. However, this tendency was mitigated for the right-handed laryngoscopists $(\mathrm{P}=0.006)$ (Table 6).

\section{DISCUSSION}

We established a hypothesis that conventional left-handed laryngoscopes are not of a reasonable design for right-handed laryngoscopists. We analyzed the usefulness of a righthanded laryngoscope in terms of securing laryngoscopic view and the distance between the teeth and blade. We found that the laryngeal view was improved, the blade-tooth distance was increased, and chances of the blade hitting the tooth decreased when a right-handed laryngoscopist used a righthanded laryngoscope.

In the present study, it was discovered that the frequency of having poor visibility equal to grade III and IV was $4.5 \%$ when a left-handed professional used a conventional laryngoscope. However, when a right-handed laryngoscope was used, the frequency increased to $22.5 \%$, indicating even poorer visibil-

Table 2. Laryngoscopist Data

\begin{tabular}{lcc}
\hline Variable & $\begin{array}{c}\text { Left-handed } \\
\text { laryngoscopists } \\
(n=18)\end{array}$ & $\begin{array}{c}\text { Right-handed } \\
\text { laryngoscopists } \\
(n=25)\end{array}$ \\
\hline R2 & $3(16.7)$ & $4(16.0)$ \\
R3 & $5(27.8)$ & $6(24.0)$ \\
R4 & $5(27.8)$ & $5(20.0)$ \\
Fellowship & $3(16.7)$ & $6(24.0)$ \\
Faculty & $2(11.1)$ & $4(16.0)$
\end{tabular}

Values are presented as number (\%). R: resident anesthesiologist.

Table 1. Patient Demographic Data

\begin{tabular}{lcc}
\hline Variable & $\begin{array}{c}\text { Laryngoscopy performed by } \\
\text { left-handed laryngoscopists }(\mathrm{n}=89)\end{array}$ & $\begin{array}{c}\text { Laryngoscopy performed by } \\
\text { right-handed laryngoscopists }(\mathrm{n}=75)\end{array}$ \\
\hline Age $(\mathrm{yr})$ & $50.1 \pm 28.0$ & $46.1 \pm 15.2$ \\
Gender $(\mathrm{M} / \mathrm{F})$ & $28(31.5) / 61(68.5)$ & $22(29.3) / 53(70.7)$ \\
Height $(\mathrm{m})$ & $1.60 \pm 0.08$ & $1.62 \pm 0.09$ \\
Weight $(\mathrm{kg})$ & $59.90 \pm 10.68$ & $60.88 \pm 9.25$ \\
BMl $\left(\mathrm{kg} / \mathrm{m}^{2}\right)$ & $23.44 \pm 3.82$ & $23.23 \pm 3.53$ \\
\hline
\end{tabular}

Values are presented as mean \pm SD or number (\%). BMI: body mass index. 
Table 3. Comparisons of Laryngoscopic Scales between Conventional and Right-handed Laryngoscopes

\begin{tabular}{|c|c|c|c|c|c|c|}
\hline \multirow[b]{2}{*}{ Variable } & \multicolumn{3}{|c|}{ Blade-tooth distance $(\mathrm{mm})$} & \multicolumn{3}{|c|}{ Contact to teeth } \\
\hline & $\begin{array}{l}\text { Conventional } \\
\text { laryngoscope }\end{array}$ & $\begin{array}{l}\text { Right-handed } \\
\text { laryngoscope }\end{array}$ & $P$ value & $\begin{array}{l}\text { Conventional } \\
\text { laryngoscope }\end{array}$ & $\begin{array}{l}\text { Right-handed } \\
\text { laryngoscope }\end{array}$ & $P$ value \\
\hline $\begin{array}{l}\text { Laryngoscopies performed by } \\
\text { left-handed laryngoscopists ( } n=89 \text { ) }\end{array}$ & $4.1 \pm 2.6$ & $2.1 \pm 1.7$ & $<0.001$ & $4(4.5)$ & $16(18.0)$ & 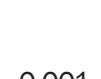 \\
\hline $\begin{array}{l}\text { Laryngoscopies performed by } \\
\text { right-handed laryngoscopists }(n=75)\end{array}$ & $5.0 \pm 3.5$ & $5.7 \pm 3.7$ & $<0.001$ & $3(4.0)$ & $1(1.3)$ & 0.001 \\
\hline
\end{tabular}

Table 4. Comparisons of Laryngoscopic View Grade between Conventional and Right-handed Laryngoscopes

\begin{tabular}{|c|c|c|c|c|c|c|c|c|c|}
\hline \multirow{2}{*}{ Variable } & \multicolumn{4}{|c|}{ Conventional laryngoscope } & \multicolumn{4}{|c|}{ Right-handed laryngoscope } & \multirow{2}{*}{$P$ value } \\
\hline & I & $\|$ & III & IV & I & II & III & IV & \\
\hline $\begin{array}{l}\text { Laryngoscopies performed by left-handed } \\
\text { laryngoscopists }(n=89)\end{array}$ & $45(50.6)$ & $40(44.9)$ & $4(4.5)$ & $0(0)$ & $35(39.3)$ & $34(38.2)$ & $20(22.5)$ & $0(0)$ & \\
\hline $\begin{array}{l}\text { Laryngoscopies performed by right-handed } \\
\text { laryngoscopists }(n=75)\end{array}$ & $29(38.7)$ & $37(49.3)$ & $9(12.0)$ & $0(0)$ & $38(50.7)$ & $34(45.3)$ & $3(4.0)$ & $0(0)$ & 0.005 \\
\hline
\end{tabular}

Values are presented as number (\%).

Table 5. Comparisons of the Improvement in Laryngoscopic View between Left-handed and Right-handed Laryngoscopists

\begin{tabular}{|c|c|c|c|c|}
\hline \multirow[b]{2}{*}{ Variable } & \multicolumn{3}{|c|}{ Grade of laryngoscopic view } & \multirow[b]{2}{*}{$P$ value } \\
\hline & $\begin{array}{l}\text { Conventional } \\
\text { > Right-handed }\end{array}$ & $\begin{array}{l}\text { Conventional } \\
=\text { Right-handed }\end{array}$ & $\begin{array}{l}\text { Conventional } \\
<\text { Right-handed }\end{array}$ & \\
\hline $\begin{array}{l}\text { Laryngoscopies performed by left-handed } \\
\text { laryngoscopists }(n=89)\end{array}$ & $1(1.1)$ & $61(86.5)$ & $27(30.3)$ & \multirow{2}{*}{$<0.001$} \\
\hline $\begin{array}{l}\text { Laryngoscopies performed by right-handed } \\
\text { laryngoscopists }(n=75)\end{array}$ & $17(22.7)$ & $56(74.7)$ & $2(2.7)$ & \\
\hline
\end{tabular}

Values are presented as number (\%). A high laryngoscopic grade indicates a poor laryngoscopic view by the Cormach-Lehane grade scale.

Table 6. Preference of Laryngoscopists

\begin{tabular}{|c|c|c|c|c|}
\hline \multirow{2}{*}{ Variable } & \multicolumn{3}{|c|}{ Frequency of the feeling of easier handling } & \multirow{2}{*}{$P$ value } \\
\hline & Conventional laryngoscope & Right-handed laryngoscope & No difference & \\
\hline $\begin{array}{l}\text { Laryngoscopies performed by } \\
\text { left-handed laryngoscopists }(n=89)\end{array}$ & 83 (93.3) & $0(0)$ & $6(6.7)$ & \multirow{2}{*}{0.006} \\
\hline $\begin{array}{l}\text { Laryngoscopies performed by } \\
\text { right-handed laryngoscopists }(n=75)\end{array}$ & $58(77.3)$ & $3(4)$ & $14(18.7)$ & \\
\hline
\end{tabular}

ity. However, the tests with a right-handed operator showed the opposite tendency, with the frequency of having poor visibility being $12 \%$ when a conventional laryngoscope was used and $4 \%$ when a right-handed laryngoscope was used. This result was consistent with those from surveys on the frequency of improvement or worsening of laryngoscope visibility. Those results can be attributed to the fact that when a left-handed operator uses a right-handed laryngoscope, his/ her right hand lacks the required refinement in control and strength to handle the blade compared with his/her left hand. Consequently, the blade tip fails to position on the vallecula precisely or the jaw is not lifted sufficiently. Similarly, when a right-handed operator uses a right-handed laryngoscope, the superior control and strength of the right hand could contribute to the precise positioning of the blade tip on the vallecula and sufficient lifting of the jaw. This explanation is supported 
by better laryngoscopic views being provided by a left-handed laryngoscopist than a right-handed laryngoscopist when a conventional laryngoscope was used (the incidence of poor view was $4.5 \%$ vs. $12 \%$ for left-handed and right-handed laryngoscopists, respectively).

We analyzed the cases in which direct contact between the blade and upper tooth was inevitable during glottic exposure, such that the actual blade-tooth distance was $0 \mathrm{~mm}$. That direct contact between the blade and upper tooth during laryngoscopy was inevitable indicates that direct pressure must inevitably be applied by the blade to the upper tooth. As most dental injuries related with anesthesia are caused by friction or by direct contact of the upper teeth with a hard blade during rigid laryngoscopy, it appears that the shorter the blade-tooth distance, the more likely that the blade will be in contact with the upper teeth, and the more frequently should upper dental injuries be sustained. Thus, in the present study, we believe the blade-tooth distance and chance of hitting the tooth provide an objective estimation of the potential for dental trauma during laryngoscopy. The frequency of contacts between the teeth and blade decreased when a left-handed operator used a conventional laryngoscope and when a right-handed operator used a right-handed laryngoscope. Considering that dental trauma is the most common complication of general anesthesia with an incidence as high as $12.1 \%[10,11]$, and is the largest single reason for successful malpractice claims against anesthesiologists [12], reducing the possibility and/or severity of dental trauma during laryngoscopy is an important goal. The results of the current study may contribute to achieve that goal.

The analysis of preference of laryngoscopists revealed that most of the left-handed operators believed that the conventional laryngoscope was more convenient to use (93.3\%), and none of them felt comfortable using a right-handed device. This likely that, for a left-handed person, using a right-handed laryngoscope may result in insufficient control and strength over the direction and depth of the laryngoscopic blade, resulting in poor visibility and increased discomfort for the operators. Conversely, the proportion of responses stating that the conventional left-handed laryngoscope was more convenient decreased to $77.3 \%$ with the right-handed operators, whereas the ratio of responses stating that the right-handed laryngoscope was more convenient increased to $4 \%$. The latter value was relatively higher than what was noted with the left-handed operators possibly because the strength and control were superior with the right-hand. However, more than three-fourths of right-handed laryngoscopists still replied that conventional left-handed laryngoscope was easier to handle with than righ-handed laryngoscope. This seems to be attributed to the cognitive aspect of right-handed larygoscopist who already was used to left-handed laryngoscope and the intuitive preference to what people are accustomed to.

To ensure that all the participants felt comfortable with the right-handed laryngoscopes before tests were conducted with actual patients, approximately 10 practice rounds were performed using a right-handed laryngoscope on a manikin. It would certainly be difficult for anesthetists to be accustomed to the new, right-handed device after years or even decades of practice with the left-handed device. A good laryngoscopy technique depends on the prior experience of the laryngoscopist. Some literature has discussed what constitutes sufficient technical experience to guarantee the adequacy of centers for teaching neophyte anesthesia professionals. For example, in a study of non-anesthesiologists learning tracheal intubation using normal subjects, 47 attempts at intubation were required before a trainee could be predicted to have a $90 \%$ probability of a competent execution of intubation [13]. In neophyte anesthesia residents, a $90 \%$ rate of adequate technical performance was achieved after 57 attempts [14]. Thus, the amount of practice with right-handed laryngoscopes in our study may not have been sufficient prior to the participation in the tests. However, the previous studies do not apply to the problems presented in our research. For neophytes, properly insertion and lifting of the laryngoscope require both knowledge and physical execution of that knowledge. However, the residents and faculty in our study had already mastered the cognitive aspects of tracheal intubation. Therefore, the barriers to successful tracheal intubation encountered by residents or faculty anesthesiologists are different than the problems facing neophytes. Nevertheless, we expect that additional sufficient rounds of practice with manikin would yield even more meaningful results. If the experimental group was not anesthesiologists, but medical students or paramedical novices, we would have more meaningful study results. In fact we are planning to have a follow up study on medical students and paramedical novices.

There are several limitations in this study. First, we did not analyze the ease of tracheal intubation aside from analyzing 
the improvement of laryngoscopic view and the blade-tooth distance by using dominant handed laryngoscope. It is important to measure the ease of tracheal intubation because the improvement of laryngoscopic view and the increase in blade-tooth distance do not always coincide with the ease of tracheal intubation, and pushing the tube into the trachea with non-dominant hand may result in worse success rate of intubation. Henceforth, further study awaits regarding the comfortableness, easiness, and the duration of tracheal intubation by non-dominant hand. However, it is considered relatively easier to push the tube into the trachea comparing with securing better laryngoscopic view, and pushing the tube into the trachea with non-dominant hand may not be as difficult if it is with better laryngoscopic view. Thus, we focused on securing laryngoscopic view and the ease of laryngoscopic manipulation in this study. Second, there might be potential bias as the operators were aware of the purpose of this study, and the graders of vocal cord view were the identical people who performed laryngoscopy. We designed this study in this way because the person who operated laryngoscopy would evaluate the grade of glottis view most accurately. We admit that this kind of study could not be done blind.

In conclusion, our results indicate that using dominant hand to hold laryngoscope can achieve better view of vocal cords and less frequency of contact between the blade and the upper teeth than using non-dominant hand.

\section{ACKNOWLEDGMENTS}

This study protocol was registered in the Clinical Research information service Republic of Korea (CRiS no. КCT0001154).

\section{REFERENCES}

1. Rosenblatt WH, Sukhupragarn W. Airway management. In: Clin- ical Anesthesia. 7th ed. Edited by Barash PG, Cullen BF, Stoelting RK, Cahalan MK, Stock MC, Ortega R: Philadelphia, Lippincott Williams \& Wilkins. 2013, pp 776-7.

2. Hawkins TJ. The left-handed laryngoscope. Anaesthesia 1999; 54: 1024 .

3. Lee J, Choi JH, Lee YK, Kim ES, Kwon OK, Hastings RH. The Callander laryngoscope blade modification is associated with a decreased risk of dental contact. Can J Anaesth 2004; 51: 181-4.

4. Lee J, Kim BS, Chang HW, Lee Y. The usefulness of the modified laryngoscopic blade for training novice laryngoscopists. J Clin Anesth 2005; 17: 334-8.

5. Elder J, Waisel DB. Case report of the one-armed anesthesiology resident. J Clin Anesth 2004; 16: 445-8.

6. Das Adhikary S, Venkatesan T, Mohanty S, Ponniah M. Difficult laryngoscopy made easy with the use of left-hand laryngoscope blade. Br J Anaesth 2007; 98: 141-4.

7. Rajeev S, Panda NB, Panda N, Batra YK. Left handed laryngoscope: a new look at an old instrument. Acta Anaesthesiol Belg 2008; 59: 139-40.

8. Watanabe S, Suga A, Asakura N, Takeshima R, Kimura T, Taguchi $\mathrm{N}$, et al. Determination of the distance between the laryngoscope blade and the upper incisors during direct laryngoscopy: comparisons of a curved, an angulated straight, and two straight blades. Anesth Analg 1994; 79: 638-41.

9. Cormack RS, Lehane J. Difficult tracheal intubation in obstetrics. Anaesthesia 1984; 39: 1105-11.

10. Rosenberg MB. Anesthesia-induced dental injury. Int Anesthesiol Clin 1989; 27: 120-5.

11. Chen JJ, Susetio L, Chao CC. Oral complications associated with endotracheal general anesthesia. Ma Zui Xue Za Zhi 1990; 28: 163-9.

12. Owen H, Waddell-Smith I. Dental trauma associated with anaesthesia. Anaesth Intensive Care 2000; 28: 133-45.

13. Mulcaster JT, Mills J, Hung OR, MacQuarrie K, Law JA, Pytka S, et al. Laryngoscopic intubation: learning and performance. Anesthesiology 2003; 98: 23-7.

14. Konrad C, Schüpfer G, Wietlisbach M, Gerber H. Learning manual skills in anesthesiology: is there a recommended number of cases for anesthetic procedures? Anesth Analg 1998; 86: 635-9. 